\title{
Auto-apprentissage et TICE
}

Le programme SEMIK en Allemagne

Self-learning and ICT - the Semik project in Germany

Autoaprendizaje y TICE. El proyecto SEMIK en Alemania

\section{Sandrine Lamer}

\section{OpenEdition}

\section{Journals}

Édition électronique

URL : https://journals.openedition.org/ries/1862

DOI : 10.4000/ries. 1862

ISSN : 2261-4265

\section{Éditeur}

France Education international

\section{Édition imprimée}

Date de publication : 1 avril 2002

Pagination : $53-63$

ISBN : 2-84520-553-8

ISSN : $1254-4590$

Référence électronique

Sandrine Lamer, «Auto-apprentissage et TICE », Revue internationale d'éducation de Sèvres [En ligne], 29 | avril 2002, mis en ligne le 01 avril 2005, consulté le 05 juillet 2021. URL : http:// journals.openedition.org/ries/1862; DOI : https://doi.org/10.4000/ries.1862 


\title{
Auto-apprentissage et TICE
}

\section{Le programme SEMIK ${ }^{1}$ en Allemagne}

\author{
Sandrine Lamer
}

«Il n'est plus possible que ce nouveau mode d'existence (lié à l'omniprésence médiatique) n'entraîne pas des conséquences profondes sur nos manières de penser, de réagir, de nous comporter. Il intervient dans la pratique professionnelle des élèves, et aussi des enseignants. Il transforme les façons de travailler comme les représentations du métier. (...) Les effets de génération tendent à s'estomper : tout le monde est pris dans le maelström ${ }^{2}$.

La toute-puissance de l'omniprésence médiatique suscite de nombreuses controverses. Un constat cependant se généralise : il est nécessaire non pas d'éviter les nouveaux médias mais au contraire de mettre en place des méthodes pour les utiliser dans l'enseignement. L'exigence des temps modernes s'oriente vers le lifelong learning et par là-même, vers les auto-apprentissages. L'école se doit de préparer les élèves à cet avenir afin de leur donner toutes les chances sur un marché du travail en pleine évolution. C'est sur cette base de réflexion que le projet pilote fédéral SEMIK a été mis en place à l'initiative de l'État libre ${ }^{3}$ de Saxe (RFA), qui en est le coordinateur ${ }^{4}$. Le modèle saxon «Développement des processus d'apprentissage (essentiellement au niveau de la Sekundarstufe $2^{5}$ ) par l'intégration systématique des technologies d'information et de communication dans l'enseignement (TICE)» est un élargissement de SEMIK et

1. SEMIK : Systematische Einbeziehung von Medien, Informations- und Kommunikationstechnologien (IKT) in LehrLernprozessen (introduction systématique des médias et des nouvelles technologies de l'information et de la communication dans les processus d'enseignement et d'apprentissage).

2. Louis Porcher, Médias et enseignement, La documentation française, Paris, 1995.

3. Parmi les seize Länder allemands, deux sont un peu plus autonomes et sont appelés "États libres »: la Bavière et la Saxe.

4. Kerstin Kühner a été chargée de coordonner le projet.

5. La Sekundarstufe 2 correspond au second cycle de la scolarité en gymnasium et comprend, selon les Länder, deux à trois années d'études au-delà de la scolarité obligatoire. 
s'applique à tester dans différentes structures publiques du secondaire la possibilité d'une réduction du temps de travail en enseignement traditionnel afin de développer l'auto-apprentissage. Trois Länder se sont associés à la Saxe dans l'application de SEMIK : le Bade-Würtemberg, la Rhénanie du Nord-Westphalie et la Sarre.

Cet article n'a pour but de développer une réflexion approfondie sur le bien-fondé de la «technicisation» de l'action cognitive des élèves ou de l'apprentissage en général. Il se veut bien plus une présentation pragmatique des initiatives d'outre-Rhin pour moderniser le système éducatif en accord avec l'évolution de notre contexte.

\section{LES NOUVELLES TECHNOLOGIES ET L'ENSEIGNEMENT}

Les initiateurs du projet d'intégration de l'auto-apprentissage guidé dans le cursus scolaire sont partis d'une réflexion d'actualité : l'introduction des TIC dans l'enseignement. Les nouvelles technologies d'information et de communication font aujourd'hui partie intégrante de toute formation. Leur utilisation est banalisée depuis leur apparition dans les milieux scientifiques, industriels et économiques, mais elle s'établit aussi depuis quelques années de plus en plus systématiquement dans les méthodologies éducatives.

Tout comme l'apparition de la «culture de masse » inquiétait les intellectuels des années d'après-guerre, la télévision était « accusée de corrompre la jeunesse et de ruiner les assises culturelles de nos sociétés " ${ }^{6}$ dans les années 80 . Ce sont ensuite les nouvelles technologies qui ont fait lever les boucliers des enseignants.

Les dangers d'une "société d'information " vont en effet au-delà de difficultés techniques et touchent bien plus aux contenus de ces informations diffusées en masse. Il s'agit donc d'apprendre à les gérer afin de construire des réseaux de savoirs fondés. C'est en cela que l'école, l'un des principaux acteurs de l'éducation, se doit d'apprendre aux jeunes à utiliser les outils auxquels ils sont confrontés au quotidien et dont ils se servent sans réelle conscience de leurs atouts et limites. Autrement dit : notre société se transforme en "société de savoirs »(Wissensgesellschaft) qui doit relever le défi.

Pour apporter une réponse aux réticences des enseignants et des familles d'une part, aux dangers d'une "mal-utilisation » des sources d'information et de communication d'autre part, le gouvernement allemand a décidé de mettre à l'étude dans chaque Land de nouvelles méthodologies d'enseignement introduisant systématiquement les TICE. Dans le cadre de projets comme SEMIK, il en étudie les conséquences pour l'évolution de l'école allemande dans un avenir proche.

6. François Mariet, Laissez-les regarder la télé, Calmann-Lévy, 1989, p. 9 


\section{Le PROgRAMme SEMIK EN RÉPUblique FÉdÉrale D'Allemagne}

Le programme SEMIK est une initiative nationale de la commission fédérale des Länder (BLK) visant à élaborer de nouveaux concepts didactiques dans le secondaire en RFA. La réalité administrative de l'Allemagne fédérale a des conséquences directes sur la politique éducative. Le Bund (État fédéral) n'intervient que très peu dans le domaine culturel et en particulier scolaire, cette compétence relevant des seize Länder. Ainsi, l'éducation, que ce soit au niveau des programmes, des examens, des cursus (douze ou treize années de scolarité selon les Länder mènent à l'Abitur), n'a pas de contours uniformes.

Un projet éducatif à dimension nationale comme SEMIK prend donc un caractère d'exception et met en évidence la valeur accordée aux objectifs didactiques fixés dans le cadre de cette étude.

Tout système éducatif fait l'objet de nombreuses réflexions directement en lien avec l'évolution socioculturelle de la société. Il doit contenir des structures, des méthodes et des enseignements aptes à former de façon optimale les futurs citoyens à leur intégration dans le monde. Nous pouvons constater que l'individu aujourd'hui prend de plus en plus conscience de son champ d'action et qu'il va de ce fait de plus en plus activement à la recherche des informations dont il a besoin. L'éducation doit donc intégrer ce nouveau phénomène et prévoir la formation à une utilisation autonome des nouveaux supports.

«Dans de nombreux domaines de la société, l'exigence d'une nouvelle acception de l'éducation en tant que valeur humaine se fait sentir, valeur permettant à l'Homme d'accéder à la maturité [...], lien entre l'autodétermination et la responsabilité ${ }^{7}$. L'autodétermination et la responsabilité passent en effet par une aptitude à gérer les informations mais aussi à respecter une distance critique vis-à-vis de leur diffusion.

Il paraît judicieux d'associer auto-apprentissage et nouvelles technologies d'information et de communication dans une perspective de complémentarité inhérente. Les TIC permettent d'avoir un accès individuel au savoir, de séparer donc du groupe dans sa forme traditionnelle et de développer l'autonomie mais aussi de "partager» dans le sens communicatif du terme. Par ailleurs, leur organisation hiérarchique appelle et favorise la " mise en ordre » et la gestion des savoirs. Enfin, l'Internet ${ }^{8}$ représente un médium utilisé par plus de 100 millions de personnes et un taux d'utilisation augmentant de près

7. Klafki, W : «Schlüsselprobleme als inhaltlicher Kern internationaler Erziehung » in Bildung und Erziehung an der Schwelle zum dritten Jahrtausend. Multidisziplinäre Aspekte, Analysen, Positionen, Perspektiven. München. PimS, 1994, pp.135-161. Cité d'après le professeur Mandel dans son étude de SEMIK. Traduction : Sandrine Lamer.

8. Interconnected Networks, le lien entre tous les ordinateurs qui peuvent communiquer ensemble à partir par exemple d'une ligne téléphonique : une confédération internationale de plus de 12000 réseaux informatiques. 
de $15 \%$ par mois : il est le symbole d'une nouvelle forme de partage, de communication, de récit.

L'introduction des nouveaux médias dans l'enseignement permet d'aller à l'encontre des inclinations des jeunes et peut leur faire renouer un lien affectif avec l'apprentissage cognitif en soi.

Il est cependant nécessaire de développer un cadre de référence et des modèles pédagogiques d'utilisation des TICE respectant la déontologie de l'enseignement et les normes morales à la base de tout bon fonctionnement d'une société, par exemple : exigence de vérité, de véracité, de responsabilité, de rigueur, notions relatives à la sécurité pour la réception et la diffusion des informations, respect de la propriété intellectuelle et des libertés individuelles.

\section{Application de SEMIK dans les différents Länder}

Lancé le premier août 1998, le projet s'achèvera le 31 juillet 2003. Cet aboutissement donnera lieu à des analyses approfondies des différents bilans établis au cours de ces années dans les seize Länder. Durant la phase expérimentale, chaque Land applique une forme spécifique d'étude de terrain d'après les objectifs généraux de SEMIK.

Ces différentes applications mettent en exergue la dimension pluridisciplinaire du projet. Elle est en effet un des objectifs majeurs de la restructuration prévue du système scolaire allemand.

\section{Organisation pour la mise en application du projet pilote fédéral}

Pour les acteurs du programme, il est nécessaire que ses objectifs soient testés dans le plus grand nombre d'établissements scolaires possible.

L'organisation opérationnelle du projet se fait à deux niveaux : national et fédéral. Au niveau du Bund, une réunion annuelle a lieu chaque automne regroupant tous les participants. Ces rencontres permettent une présentation des premiers résultats obtenus, un échange d’idées sur la base de réflexions engendrées par les présentations et par les conférences tenues par des universitaires sur des thèmes liés à SEMIK. Au niveau du Land, des groupes de travail (sets) sont organisés dans chaque Land en fonction des profils scolaires (Gymnasien, Realschulen ou Mittelschulen ${ }^{9}$, distance learning, formation des maîtres, etc.). Chaque set compte plusieurs établissements.

9. Pour l'organisation du système scolaire allemand, voir Ingrid Gogolin « Une nouvelle offensive, l'évolution du système éducatif en Allemagne » in Revue internationale d'éducation - Sèvres $\mathrm{n}^{\circ} 28$, décembre 2000, pp. 33-39. 


\begin{tabular}{|c|c|c|}
\hline Projet & Land & Intitulé du projet pilote \\
\hline 1. & Bade-Wurtemberg & Programme SEMIK (cf. projet pilote en Saxe) \\
\hline 2. & Bade-Wurtemberg & Didactique d'utilisation des médias par un forum on line \\
\hline 3. & Bavière & $\begin{array}{l}\text { Développement de projets pilotes pour l'utilisation de systèmes multimé- } \\
\text { dias interactifs dans l'enseignement }\end{array}$ \\
\hline 4. & Bavière & Management du savoir à l'école et développement du système scolaire \\
\hline 5. & Berlin & $\begin{array}{l}\text { Développement, mise en place et mise à l'épreuve de matériel numérique } \\
\text { pour l'enseignement et l'apprentissage }\end{array}$ \\
\hline 6. & Berlin & $\begin{array}{l}\text { Conception de formations pour le développement d'une nouvelle culture } \\
\text { de l'apprentissage }\end{array}$ \\
\hline 7. & Brandebourg & Atelier numérique \\
\hline 8. & Brandebourg & Développement de la filière «médias et communication» au lycée. \\
\hline 9. & Brême & Ouverture de l'enseignement à l'auto-apprentissage grâce aux TICE \\
\hline 10. & Brême & $\begin{array}{l}\text { Utilisation pluridisciplinaire des ordinateurs en tant qu'outils de travail } \\
\text { dans le cadre de l'enseignement spécialisé regroupant des enfants sans han- } \\
\text { dicap et des handicapés mentaux }\end{array}$ \\
\hline 11. & Hambourg & Développement d’unités d’enseignement \\
\hline 12. & Hesse & $\begin{array}{l}\text { Nouvelles réalités d'apprentissage à l'école et dans les deuxièmes cycles de } \\
\text { formation à l'enseignement }\end{array}$ \\
\hline 13. & $\begin{array}{l}\text { Mecklembourg } \\
\text { Vorpoméranie }\end{array}$ & $\begin{array}{l}\text { Intégration des ordinateurs dans les structures d'enseignement et d'appren- } \\
\text { tissage des lycées professionnels }\end{array}$ \\
\hline 14. & $\begin{array}{l}\text { Mecklembourg } \\
\text { Vorpoméranie }\end{array}$ & $\begin{array}{l}\text { Développement de compétences méthodologiques pour l'acquisition des } \\
\text { savoirs }\end{array}$ \\
\hline 15. & $\begin{array}{l}\text { Mecklembourg } \\
\text { Vorpoméranie }\end{array}$ & Développement des curriculum et nouveaux médias \\
\hline 16. & Basse Saxe & $\begin{array}{l}\text { Culture disciplinaire et médias. Développement et mise à l'épreuve de } \\
\text { concepts dans la formation à l'enseignement }\end{array}$ \\
\hline 17. & $\begin{array}{l}\text { Rhénanie du Nord- } \\
\text { Westphalie }\end{array}$ & Programme SEMIK (cf. projet pilote en Saxe) \\
\hline 18. & $\begin{array}{l}\text { Rhénanie du Nord- } \\
\text { Westphalie }\end{array}$ & $\begin{array}{l}\text { Conception et développement de modules de communication et coopéra- } \\
\text { tion en éducation dans le cadre d'exercices d'apprentissage sur des serveurs }\end{array}$ \\
\hline 19. & $\begin{array}{l}\text { Rhénanie du Nord- } \\
\text { Westphalie }\end{array}$ & $\begin{array}{l}\text { Projet pilote d'utilisation des médias en modules grâce aux informations } \\
\text { tirées du réseau }\end{array}$ \\
\hline 20. & Rhénanie-Palatinat & $\begin{array}{l}\text { Auto-apprentissage dans l'enseignement pluridisciplinaire concernant la } \\
\text { géographie, l'éducation civique, l'économie; les mathématiques et les } \\
\text { sciences expérimentales au collège }\end{array}$ \\
\hline 21. & Sarre & Programme SEMIK (cf. projet pilote en Saxe) \\
\hline 22. & Saxe & $\begin{array}{l}\text { Titre court : } 20 \text { plus } \\
\text { Titre complet : Développement des processus d'apprentissage (essentielle- } \\
\text { ment au niveau de la Sek2) par l'intégration systématique des technologies } \\
\text { d'information et de communication dans l'enseignement (TICE) } \\
\text { Projet de coopération entre quatre Länder : la Saxe (direction du projet), le } \\
\text { Bade-Wurtemberg, la Rhénanie du Nord-Westphalie, la Sarre) }\end{array}$ \\
\hline 23. & Saxe-Anhalt & $\begin{array}{l}\text { Développement et mise à l'épreuve d'éléments de curriculums pour l'utili- } \\
\text { sation systématique des médias et des TIC au collège et lycée }\end{array}$ \\
\hline 24. & Schleswig-Holstein & $\begin{array}{l}\text { Les nouveaux médias dans la deuxième phase de la formation à l'ensei- } \\
\text { gnement }\end{array}$ \\
\hline 25. & Schleswig-Holstein & $\begin{array}{l}\text { Recherche des utilisations didactiques optimales de l'ordinateur dans } \\
\text { l'enseignement des sciences }\end{array}$ \\
\hline 26. & Thuringe & $\begin{array}{l}\text { Développement et mise à l'épreuve de concepts incluant les nouvelles tech- } \\
\text { nologies d'information et de communication dans la deuxième phase de la } \\
\text { formation à l'enseignement }\end{array}$ \\
\hline
\end{tabular}

1. Sek2= Sekundarstufe 2 . 
Des formations dirigées par des experts sont proposées aux enseignants (formation initiale et continue à l'utilisation des médias, à l'auto-évaluation et à l'évaluation de projets, etc.).

Les établissements impliqués dans le projet proposent également des formations internes au cours desquelles ils présentent leurs initiatives, échangent des unités modèles d'enseignement, ouvrent leurs cours à des observations.

Enfin, des séminaires entre coordinateurs ont également lieu à intervalles réguliers : soit à l'intérieur des sets, soit au niveau du Land pour l'élaboration des modèles de curricula, la mise au point d'outils pédagogiques et d'unités d'enseignement et la rédaction de fiches d'auto-évaluation.

Voyons pour illustration l'exemple du modèle saxon, l'État libre de Saxe jouant le rôle central d'initiateur et de directeur de SEMIK.

\section{LE MOdÈLE SAXON : « 20 Plus "}

« 20 plus » 10 est l'abréviation officielle du modèle saxon : «Développement des processus d'apprentissage (essentiellement au niveau de la «Sek2») par l'intégration systématique des TICE ». La durée expérimentale prévue pour ce projet pilote de la Commission fédérale des Länder dans l'État libre de Saxe était initialement du $1^{\mathrm{er}}$ octobre 1998 au 30 septembre 2002. Les résultats cependant ont été si positifs jusqu'alors qu'une prolongation a été accordée au Land jusqu'au 28 février 2003.

\section{Pourquoi « 20 Plus » ?}

Grâce aux phases d'auto-apprentissage, le nombre d'heures réservées à l'enseignement traditionnel doit être à long terme réduit à « 20 plus $\mathrm{X}$ » heures de cours dans le secondaire, $\mathrm{X}$ étant le nombre d'heures ou la mesure à déterminer par le projet pilote réservée aux auto-apprentissages guidés. Actuellement, les élèves du secondaire ont, en Saxe, un volume hebdomadaire de 32 à 35 heures de cours. Dans l'idéal, une réduction de ce temps d'enseignement traditionnel serait instaurée afin de recentrer l'apprentissage sur l'élève et d'autonomiser son approche du savoir.

L'objectif central consiste donc à introduire une disciplinarisation de l'utilisation des nouveaux médias et des TIC en développant les phases d'autoapprentissage dans les cursus scolaires ${ }^{11}$. Ainsi, le système éducatif serait en phase avec les nouvelles modalités d'accès au et de diffusion du savoir. Il prendrait en compte l'intérêt croissant des jeunes pour les nouveaux médias et utiliserait cet attrait à des fins propédeutiques.

10. Pour plus d'informations, consulter http://www.sn. schule.de/semik-20plus/sachsen/

11. Cf. revue Fingerzeiger, juillet 1999 


\section{De la cause à l'effet...}

Les élèves, nous l'avons dit, doivent être préparés aux nouvelles exigences de la "société de savoirs" pour leurs études et leur vie professionnelle. Cela suppose entre autres qu'ils soient capables d'utiliser les nouveaux médias pour se former leur vie durant. Le projet " 20 plus » a d'ores et déjà permis un certain nombre de modifications allant dans ce sens au niveau de l'organisation du système scolaire des établissements y participant.

L'utilisation des nouvelles technologies reste cependant au service des objectifs pédagogiques et vient compléter les sources traditionnelles et outils de travail propres au milieu scolaire. Le livre ne disparaît pas, le cartable non plus. Certaines informations peuvent néanmoins être actualisées via les TICE plus rapidement que par l'imprimé traditionnel. Il n'est pas question de virtualiser totalement l'école mais de la moderniser. Par ailleurs, grâce à " 20 plus ", l'enseignant acquiert un nouveau rôle. Il devient un médiateur et un conseiller. Il n'est plus seulement le détenteur du savoir ni le seul détenteur du savoir. Les connaissances s'acquièrent progressivement de manière interactive. Dans la pratique, cela signifie que l'enseignant travaille en problématisant le thème de son unité de cours (problemorientierter Unterricht). Il annonce le cadre d'étude, fournit les contenus de base nécessaires à une bonne compréhension du sujet, fixe les objectifs et conseille sur les méthodes de travail. Les contenus en revanche sont élaborés par les élèves avec le soutien individualisé de l'enseignant qui, grâce à l'autonomie de travail du groupe-classe, peut approfondir certaines questions selon leurs besoins personnels. Il doit s'adapter à leur personnalité et accepter la voie que ceux-ci auront choisie pour aboutir au résultat attendu, et leurs conclusions si elles s'avèrent fondées.

Les unités d'enseignement apparaissent davantage sous forme de projets, souvent pluridisciplinaires, l'informatique faisant moins l'objet d'une étude en soi que d'une utilisation systématisée de ses ressources. Des innovations comme le teamteaching ${ }^{12}$ ou l'enseignement en tandem permettent d'assurer les enseignements ouverts. On a appliqué cette nouvelle méthodologie en partie dans le cadre de l'emploi du temps donné, en partie en dehors des cours. La plupart des projets proposent, dans les huit Gymnasien participant à « 20 plus », des croisements entre disciplines impliquant l'allemand, l'anglais, les arts plastiques, la biologie, la chimie, le français, la géographie, l'histoire, l'informatique, les mathématiques, la musique et les sciences physiques, les associations de disciplines variant d'un établissement à l'autre en fonction des projets, comme le montrent les exemples suivants :

- histoire, allemand, français : «Les nouveaux médias au service d'une mémoire européenne collective », concept expérimental d'un travail binational

12. Présence de plusieurs enseignants de disciplines différentes dans le même cours pour apporter aux élèves les réponses adéquates dans les projets pluridisciplinaires. 
franco-allemand sur l'histoire des identités nationales en Europe entre 1815 et 2000 ;

- histoire, allemand, arts plastiques : " le réalisme au XIX ${ }^{\mathrm{e}}$ siècle » ;

- mathématiques, informatique : "géométrie analytique";

- biologie, histoire : « les biotopes des étangs des carrières de Schöna : évolution de la nature dans son contexte historique »;

- musique, informatique : «introduction à la composition musicale grâce aux nouvelles technologies ».

Cette méthode plus autonome de travail doit permettre aux élèves de prendre conscience des liens interdisciplinaires existants et leur faciliter l'apprentissage. On n'exige plus uniquement qu'ils soient aptes à reformuler les connaissances acquises mais, au-delà, qu'ils parviennent à développer une faculté d'analyse critique, de "management du savoir»(Wissensmanagement). Leur apprentissage porte non seulement sur les contenus, mais aussi sur la gestion du temps, des outils d'apprentissage et des ressources humaines.

En effet, un travail d'équipe élèves/enseignants est indissociable de l'autonomisation. Les élèves doivent s'entraider et profiter de leurs compétences réciproques, faire appel aux enseignants mais aussi les conseiller. Il n'est pas rare en effet que les jeunes, essentiellement au niveau de la technique, maîtrisent mieux l'outil que l'enseignant. Les rôles se trouvent inversés. Enfin, les professeurs doivent coopérer entre eux pour une optimisation de la diffusion des enseignements, en comparant par exemple les contenus des curriculums par discipline et en organisant des sessions de travail thématiques parallèles ou des projets transversaux.

\section{Première ÉVAluation DU PROJET}

Une première évaluation de SEMIK fait apparaître les différentes conclusions provisoires à l'issue des travaux réalisés dans les Länder au cours de l'année 2000.

\section{Un bilan positif}

Dans l'ensemble, les participants s'accordent à voir en cette nouvelle méthode de travail l'espoir d'une nette amélioration du système scolaire. La responsabilisation des élèves semble les placer dans une situation de défi à relever et a des effets de motivation très positifs. La qualité du cours se trouve renforcée par ces travaux de recherche plus spécifiques ainsi que cette méthode d'apprentissage conscient et actif. Par ailleurs, les apprenants comme les enseignants ont acquis une compétence dans le multimédia qu'ils apprécient grandement. Enfin, le climat de travail s'est sensiblement détendu à tous les niveaux. 


\section{Difficultés rencontrées}

Quelques difficultés ont inévitablement été rencontrées sur la base desquelles une réflexion est conduite en vue de la généralisation escomptée de cette méthode.

Le travail de préparation et de suivi que doit fournir l'enseignant est alourdi par cette méthode (pour les préparations, les permanences pour conseiller les élèves individuellement en dehors des cours...). Cette charge supplémentaire rend quelques enseignants réticents. Il faudrait donc veiller à aménager aussi leurs emplois du temps avec des heures durant lesquelles ils pourront effectuer leur travail de conseiller.

La seconde grande difficulté réside dans la gestion du matériel informatique, les enseignants d'informatique étant sollicités pour l'enseignement de leur discipline. Si les phases d'auto-apprentissage sont renforcées dans les cursus, les élèves auront par ailleurs besoin de salles d'informatique accessibles à tout moment à l'école. Il sera donc nécessaire d'aménager des espaces adéquats et d'organiser leur maintenance.

Vient ensuite la question des moyens financiers mis à disposition : qui dit ouverture des apprentissages dit nécessité de se procurer des outils (livres, logiciels, accès Internet etc.).

Enfin, tout élève n'est pas apte à travailler en autonomie même guidée ; il faut veiller à ne pas décourager les plus faibles et à proposer des conditions de travail qui ne soient pas discriminatoires.

Cinq facteurs sont essentiels pour la réussite d'un tel projet :

- l'équipement des établissements en matériel informatique ${ }^{13}$ supposant une mise à disposition de personnel expert pour la gestion de ces réseaux et du matériel, un enseignement de la discipline informatique à tous les niveaux du secondaire ;

- la flexibilité des enseignants ;

- l'intégration de ces nouvelles méthodes dans les projets d'établissements (structure/emplois du temps);

- une communication efficace entre élèves, entre enseignants et élèves, entre enseignants, entre enseignants et direction de l'établissement, entre coordinateurs et acteurs du projet;

- une planification à long terme.

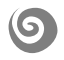

L'auto-apprentissage guidé expérimenté en Allemagne actuellement, et particulièrement en Saxe et dans les Länder qui lui sont associés, semble prendre une importance majeure pour les méthodes d'enseignement de demain.

13. Voir parallèlement le programme national « Medios » de modernisation générale sur le plan informatique des établissements scolaires allemand. 
Qui dit "auto-apprentissage » pense "nouvelles technologies». Il est préférable, certes, de rester un observateur distant et un utilisateur critique des nouveaux médias, tout comme on aborde un livre ou un journal avec prudence avant d'en vanter les contenus et la portée. C'est pourquoi il s'avère nécessaire de former les élèves à leur futur statut d'homo sapiens informaticus (celui pour lequel l'utilisation de l'outil informatique sera inhérent à tout apprentissage), succédant à celui d'homo sapiens sapiens (dont le devoir principal était l'apprentissage cognitif en soi), pour reprendre une expression du professeur K. Haefner de l'université de Brême. ${ }^{14}$

La devise du projet SEMIK est donc : soyons intelligemment actifs dans ce phénomène de roulis qui semble nous envahir et tirons-en consciemment le maximum de profit.

Si « la vérité est que l'automatisme est inhérent à tous les processus quelle qu'en soit l'origine ${ }^{15}$ ", l'Homme est certes "soumis » à ces processus automatiques d'omniprésence médiatique, mais il peut, à l'intérieur de ceuxci, "s'affirmer par l'action ». Puisque ces nouveaux moyens d'enseignement et d'apprentissage s'offrent à nous, utilisons-les le plus judicieusement possible!

\section{Bibliographie}

LANDESINSTITUT FÜR LEHRERFORTBILDUNG, LEHRERWEITERBILDUNG und UNTERRICHTSFORSCHUNG VON SACHSEN-ANHALT, (1998): Wege zur Medienkompetenz - Ein Gesamtkonzept der schulischen Medienerziehung mit Anregungen für die Unterrichtsgestaltung. Berlin. Pac-tee.

MANDEL H., KRUPPA K. und HENSE J., (2001): Zwischenbericht der wissenschaftlichen Programmbegleitung und zentralen Evaluation des BLKProgramms SEMIK für das Kalenderjahr 2000, Ludwig Maximilians Universität. München. Institut für empirische Pädagogik und pädagogische Psychologie.

GRÄSEL C., MANDL H., MANHARDT P., KRUPPA K., (2000) : Das BLKProgramm "Systematische Einbeziehung von Medien, Informations- und Kommunikationstechnologien in schulische Lehr- und Lernprozesse ", Unterrichtswissenschaft, 28, pp.127-143

14. cf. Prof. K. Haefner, discours pour le groupe de travail « formation fondamentale aux techniques de l'information " (Arbeitsgruppe informationstechnische Grundbildung), "Wie soll homo sapiens informaticus erzogen werden ? ", formation continue pour enseignants, in revue Engagement, 8/1998.

15. Hannah Arendt, La crise de la culture, Gallimard, 1972, p. 219. 
MANDEL H., REINMANN-ROTHMEIER G., GRÄSEL C., (1998) : «Gutachten zur Vorbereitung des Programms Systematische Einbeziehung von Medien, Informations- und Kommunikationstechnologien in schulische Lehr- und Lernprozesse ", in Bund-Länder-Kommission für Bildungsplanung und Forschungsforderung, Bonn, Heft 66.

WEINRICH F. und SCHULZ-ZANDER R., (2000): "Schulen ans NetzErgebnisse der bundesweiten Evaluation", Zeitschrift für Erziehungswissenschaft, 3, 577-593

Voir aussi :

www.fwu.de/semik

www.sn.schule.de/semik-20plus/sachsen/ 